Meta

Journal des traducteurs

Translators' Journal

\title{
Territorialité et extraterritorialité de la traduction du droit
}

\section{Sylvie Monjean-Decaudin}

Volume 55, numéro 4, décembre 2010

De la localisation à la délocalisation - le facteur local en traduction

From Localization to Delocalization - The Local Factor in Translation

URI : https://id.erudit.org/iderudit/045686ar

DOI : https://doi.org/10.7202/045686ar

Aller au sommaire du numéro

\section{Éditeur(s)}

Les Presses de l'Université de Montréal

\section{ISSN}

0026-0452 (imprimé)

1492-1421 (numérique)

Découvrir la revue

Citer cet article

Monjean-Decaudin, S. (2010). Territorialité et extraterritorialité de la traduction du droit. Meta, 55(4), 693-711. https://doi.org/10.7202/045686ar

\section{Résumé de l'article}

Langues, droits et territoires constituent un tout indissociable que la traduction du droit ne peut éluder. Les langues et les droits présentent à la fois une unicité et une diversité, une égalité et une hiérarchie selon l'angle de vision. La traduction non territorialisée correspond à une traduction verticale dans un contexte institutionnel de droit international ou régional négocié.

L'équivalence traductionnelle se veut dès lors uniformisante afin de faciliter une interprétation uniforme d'un droit unique énoncé dans une multitude de versions linguistiques. L’Union européenne constitue, en ce sens, un paradigme. La traduction territorialisée correspond à une traduction horizontale réalisée dans les divers contextes de droit. Destinée, entre autres, à la connaissance d'un droit autre ou à la reconnaissance du droit de l'autre, voire à l'administration de justice, cette traduction présente des caractéristiques culturelles marquées. L'équivalence traductionnelle se veut dès lors localisée car empreinte d'une culture d'origine. Face au risque d'enfermement, le traducteur doit se prémunir contre tout juricentrisme. Cependant, peut-il éviter qu'un hégémonisme linguistique, juridique et territorial ne mène à une équivalence traductionnelle ?
Ce document est protégé par la loi sur le droit d'auteur. L'utilisation des services d’Érudit (y compris la reproduction) est assujettie à sa politique d'utilisation que vous pouvez consulter en ligne.

https://apropos.erudit.org/fr/usagers/politique-dutilisation/ 


\title{
Territorialité et extraterritorialité de la traduction du droit
}

\author{
SYLVIE MONJEAN-DECAUDIN \\ Université Paris Ouest Nanterre La Défense, Paris, France \\ sylvie.monjean@u-paris10.fr
}

\begin{abstract}
RÉSUMÉ
Langues, droits et territoires constituent un tout indissociable que la traduction du droit ne peut éluder. Les langues et les droits présentent à la fois une unicité et une diversité, une égalité et une hiérarchie selon l'angle de vision. La traduction non territorialisée correspond à une traduction verticale dans un contexte institutionnel de droit international ou régional négocié. L'équivalence traductionnelle se veut dès lors uniformisante afin de faciliter une interprétation uniforme d'un droit unique énoncé dans une multitude de versions linguistiques. L'Union européenne constitue, en ce sens, un paradigme. La traduction territorialisée correspond à une traduction horizontale réalisée dans les divers contextes de droit. Destinée, entre autres, à la connaissance d'un droit autre ou à la reconnaissance du droit de l'autre, voire à l'administration de justice, cette traduction présente des caractéristiques culturelles marquées. L'équivalence traductionnelle se veut dès lors localisée car empreinte d'une culture d'origine. Face au risque d'enfermement, le traducteur doit se prémunir contre tout juricentrisme. Cependant, peut-il éviter qu'un hégémonisme linguistique, juridique et territorial ne mène à une équivalence traductionnelle?
\end{abstract}

\section{ABSTRACT}

Languages, law and territories form one inseparable whole, which has to be taken as such by legal translation. Languages and law present at once unity and plurality, equality and hierarchy, according to the angle of vision. A case in point is "translation unbound by territory," i.e., vertical translation in the institutional context of negotiated international or regional law. Translation equivalence is then established in order to achieve uniform interpretation of one unique law expressed in various linguistic versions. The European Union is paradigmatic of such a case. At the opposite, "territory-bound translation" entails horizontal translation realized in various legal contexts. It may serve various purposes, among which knowledge of the other law or recognition of the right of others, or even the administration of justice, and exhibits marked cultural characteristics. Translation equivalence is then subject to the local factor since it has to take the original culture into account. Faced with the risk of communication failure, the translator must be wary of acting as if his/her legal system were universal. However, can he/she prevent the search for translation equivalence to be guided by linguistic, legal and territorial imperialism?

\section{MOTS-CLÉS/KEYWORDS}

langues, droits, territoires, traduction horizontale, traduction verticale languages, law, territory, horizontal translation, vertical translation 
La traduction juridique répond à des nécessités pratiques impérieuses qu'il est possible d'envisager successivement du côté de la théorie générale du droit, puis - sur une pente encore plus philosophique - du côté de la linguistique et de la philosophie du langage.

(Terré 1986: 347)

Notre sujet prend racine dans le rapport langue(s), droit(s) et territoire(s). Nos propos introductifs tenteront une définition de ces concepts et une mise en relief de leur interférence, préliminaire indispensable avant la présentation de la territorialité et de l'extraterritorialité de la traduction du droit. Cet article ne prétend pas couvrir l'exhaustivité du sujet mais vise à apporter quelques éléments de réflexion sur la manière dont peut être appréhendée la traduction du droit selon les contextes juridiques, linguistiques et territoriaux.

La langue et le droit constituent le substrat principal qui nourrit le processus de la traduction juridique. Le territoire se profile en toile de fond dans l'analyse du binôme «langue-droit». Ce binôme présente diverses facettes que nous tenterons de dévoiler au regard de leurs identité, diversité, égalité et supériorité.

Les langues comme les droits se définissent par leur identité avant de se caractériser par leur diversité. La langue apparaît «comme l'ensemble des moyens d'expression, comme un code commun à l'ensemble des individus appartenant à une même communauté linguistique» (Dubois, Giacomo, et al. 2001: 93). Au sein de chaque communauté, la langue constitue «le creuset où se sculpte l'histoire d'un peuple. Les mots qui la composent et le discours qui l'exprime traduisent les valeurs d'un patrimoine commun» (Gémar 2002: 164). En ce sens, la langue constitue un produit social ${ }^{1}$. La langue en usage dans une société donnée reflète la culture de sa population (Lévi-Strauss 1958: 78). La relation de cause à effet entre la culture au sens large et la langue traduit les liens consubstantiels qu'elles entretiennent ${ }^{2}$. La multitude de langues exprimées - plus de 6000 dans un monde post-babélien non monolingue - témoigne d'une pluralité de cultures qui sont localisées géographiquement. Forgeant un lien d'appartenance d'un groupe social à un territoire, les langues «sont toutes dans un premier temps territorialisées» (Guillorel et Koubi 1999: 8).

«Définir le droit d'une manière homogène et définitive paraît impossible» (Bergel 2004: 19), là n'est pas notre intention. Notre but vise à identifier le droit, à en dégager la quintessence pertinente au regard de notre étude. Les auteurs s'entendent, d'une façon quasiment unanime, pour définir le droit comme l'ensemble des règles régissant la vie en société (Terré 2006: 3; Cornu 2007: 333; Bergel 2004: 2). Autrement dit, les règles de droit ${ }^{3}$ encadrent la vie de l'homme en société, elles sont issues de la culture, et non de la nature (Terré 2006: 33). Les comportements sociaux influencent le juridique (Terré 2006: 30), le social fait évoluer le juridique, ce qui en fait un phénomène social (Cornu 2005: 4). Ainsi, le «droit s’inscrit inéluctablement en une culture juridique (et, par son truchement, en une tradition juridique). Il est prescrit par elle» (Legrand 2006: 5-6). Dépendant de l'histoire, de l'environnement humain, social, économique..., des choix idéologiques (Bergel 2004:2), cette culture juridique émane de la culture tout court, comme "grammaire génératrice, tout en contribuant à la conforter» (Legrand 2006: 6).

En outre, la notion de droit d'un pays renferme une dimension spatiale et temporelle. Le droit existe à un moment donné et sur un territoire délimité (Bergel 2004: 
15 et 27). Même s'il ne s'applique pas exclusivement à un seul territoire, le droit est le produit d'une culture territorialisée exprimée, fruit «d'une interaction spatiotemporelle entre individus co-existant en une communauté identifiable» (Legrand 2006: 8).

Par conséquent, la multiplicité des langues et des droits a conduit à une classification en familles, mettant au jour des relations de parenté.

Tant pour le droit que pour la langue, la classification naît de la géographie et de la génétique. Pour la langue, «la partie de la dialectologie qui s'occupe de localiser les unes par rapport aux autres les variations des langues s'appelle le plus souvent géographie linguistique» (Dubois, Giacomo, et al. 2001: 218). Des atlas sont établis afin de visualiser les variations géographiques de la langue. En outre, en vue d'une classification, il est admis que deux ou plusieurs langues appartiennent à la même famille dès lors qu'elles sont apparentées génétiquement, c'est-à-dire quand tout laisse à penser qu'elles se sont développées à partir d'une origine commune ${ }^{4}$. Généralement, l'on réserve la dénomination de famille de langues à l'ensemble formé par toutes les langues connues de même origine (Breton 2008: 14). Par exemple, "parmi les familles de langues, celle qui a été la mieux établie par F. Bopp et dont l'étude a servi de modèle à toutes les recherches de la grammaire comparée est la famille des langues indo-européennes» (Dubois, Giacomo, et al. 2001: 197).

En droit, il s'avère difficile de se retrouver dans les ensembles juridiques du monde. Et c'est une grande question de l'ordre méthodologique de savoir s'il faut raisonner en "génétique juridique» ou se contenter d'organiser plus prudemment, plus modestement, une "géographie juridique». La première perspective est sans doute trop ambitieuse, la seconde trop modeste (Legeais 2008: 87). La notion de «système» (Terré 2006: 50), ont pensé beaucoup de juristes, permet une démarche intermédiaire (Legeais 2008: 87).

Ainsi, «classer les systèmes de droit c'est rechercher si certains d'entre eux présentent des traits communs suffisamment importants et nombreux pour être réunis dans une même catégorie, une même famille, un même groupe» (Legeais 2008: 88). De nombreuses classifications ont été avancées, et les transformations du monde du $\mathrm{xx}^{\mathrm{e}}$ siècle ont remis en question la classification du professeur René David ${ }^{5}$. Partant des critères de la technique et de l'idéologie $e^{6}$, il opère une classification en trois familles principales: la famille romano-germanique, la famille des systèmes de common law et la famille des systèmes de droit socialiste. Aujourd'hui, une nouvelle approche est recherchée ${ }^{7}$. Le comparatiste Raymond Legeais propose une classification en deux groupes des systèmes de droit: celui des États à longue tradition juridique et celui des États sans tradition juridique ou des États subordonnant le droit, soit à une religion, soit à une idéologie, ce qui ne permet pas de les retenir comme États de droit (Legeais 2008: 93).

La multiplicité des langues et des droits et leur classification posent la question de leur hiérarchisation. Une langue équivaut-elle à une autre langue, un droit vaut-il un autre droit? Dans l'absolu (au titre de l'égalité des cultures), la réponse serait positive, mais en réalité, une hiérarchisation existe.

La théorie linguistique générale constate une égalité. En effet, «toutes les langues sont bâties sur le même modèle. Certes, la correspondance ainsi établie ne postule pas l'isomorphisme des langues, qui ne coïncident jamais point par point» (Dubois, Giacomo, et al. 2001 : 501). Cependant, «la grammaire générale consiste à définir un 
ensemble de règles considérées comme des universaux du langage dont sont déduites les règles particulières à chaque langue» (Dubois, Giacomo, et al. 2001: 501). Ces universaux du langage correspondent aux similarités existant dans toutes les langues du monde ${ }^{8}$. En outre, les règles fondamentales du fonctionnement du langage relèvent d'une compétence universelle, sous-tendue par une organisation corticale identique.

De même, la relation d'égalité entre les différents droits ne devrait pas se poser. Pourtant, si les classifications des systèmes de droit «ne sont que des méthodes d'approche où il n'y a pas lieu de voir une recherche de hiérarchie quelconque» (Legeais 2008: 88), force est de constater que certains sont dits "grands systèmes de droit»". Ceci s'explique non pas "par leur importance en eux-mêmes, mais parce qu'ils permettent de mieux comprendre les autres" (Legeais 2008: 2). "Comme il y a des langues plus parlées que d'autres, même si toute langue a une valeur humaine infinie, il y a des systèmes de droit auxquels on doit se référer particulièrement et qui sont comme des clefs de la vie juridique du monde» (Legeais 2008: 2). Pour des raisons, entre autres, historiques, politiques, économiques, des influences et des extensions de tel ou tel système juridique se sont exercées dans le monde ${ }^{10}$.

Dans les temps modernes, les diverses formes du colonialisme, assorties d'ailleurs d'aménagements fort différents suivant les politiques, les prestiges, les conquêtes militaires, les influences universitaires, les pratiques commerciales, ont favorisé un rapprochement des droits. (Terré 2006: 59)

Ces transferts plus ou moins étendus de droits ont créé des mouvements utopiques d'unification, que la raison a ramenés à une harmonisation des systèmes, voie réaliste satisfaisante conciliant impératifs de rapprochement et attachement aux cultures juridiques nationales.

La mondialisation semble inéluctable et si elle n'implique pas obligatoirement un phénomène de conflits des droits en présence, elle s'inscrit plutôt dans une fusion librement consentie des divers systèmes juridiques par une harmonisation des droits (Terré 2006: 152).

La mondialisation procède également à des transferts linguistiques. Selon l'hypothèse, dite «loi d'interférence», Toury (1995: 275) établit que «le processus de transfert d'une langue à l'autre a tendance à s'accompagner d'interférences linguistiques ». Celles-ci sont inhérentes, d'après lui, au processus mental de la traduction, mais le degré de tolérance à leur égard dépend du "prestige» des langues en présence: les interférences les plus manifestes se trouvent dans les textes traduits d'une langue / culture «majeure» vers une langue / culture «mineure» ou «faible».

Ce constat rejoint les études menées par deux linguistes philologues, Gianfranco Folena (1991) et Benvenuto Terracini (1957), sur la traduction de la littérature médiévale $^{11}$. Plus précisément, Folena procède à la distinction entre traduction verticale et traduction horizontale. Il qualifie de traduction verticale, la traduction effectuée du latin vers une langue vulgaire (étant entendu ici que la dénomination «langue vulgaire» se réfère principalement aux langues romanes, qui était opposée au latin reconnu comme langue savante) et de traduction horizontale, celle réalisée entre deux langues vulgaires.

Ainsi, la traduction est verticale lorsqu'elle est réalisée d'une langue de statut culturel supérieur vers une autre de statut culturel inférieur et horizontale lorsqu'elle est effectuée entre deux langues de statut égal. 
La distinction opérée pour la traduction médiévale ne vaudrait-elle pas pour la traduction du droit? De même, ne pouvons-nous pas constater, aujourd'hui, la coexistence de deux types de traduction sur le territoire de l'Union européenne? D'une part, lorsque le droit de l'Union est traduit afin de s'insérer, directement ou indirectement, dans les ordres juridiques des États membres, la traduction est verticale. Sa verticalité révèle la hiérarchie de la norme qu'elle énonce, dans toutes les langues officielles, à destination des États membres et la force contraignante de son contenu. D'autre part, lorsque la traduction est réalisée au sein d'un État, elle est horizontale. Son horizontalité révèle une communication établie entre deux droits et deux langues de statut égal. La traduction horizontale est rattachée à une culture nationale territorialisée.

Forte de cette distinction, notre étude vise à présenter les caractéristiques propres à la traduction du droit, d'une part, non territorialisée, et, d'autre part, territorialisée, ainsi qu'à démontrer comment la notion d'équivalence traductionnelle diffère dans chaque cas.

\section{La traduction du droit non territorialisée}

Entre l'«individualisme international qui considère comme intangible la souveraineté nationale» et «le fédéralisme d'États qui se subordonne à un super-État doté d'une souveraineté territoriale propre», Robert Schuman définissait en 1953 la supranationalité qu'il proposait à la Communauté européenne d'adopter ${ }^{12}$. Cette supranationalité s'est construite et dès la signature du traité de Rome du 25 mars 1957, le principe du multilinguisme intégral a été adopté. Le multilinguisme signifie qu’il ne peut être fait recours à une langue unique. L'Union européenne constitue un paradigme en tant qu'expression plurilingue d'un droit unique.

En application de ce principe, aujourd'hui, le droit de l'Union européenne s'énonce dans vingt-trois langues officielles. Ceci est une exception car généralement le droit de création internationale ne peut s'élaborer ni s'exprimer dans toutes les langues des États signataires d'une convention ou d'un traité international. Il en résulte qu'un choix est opéré pour déterminer les langues officielles parmi quelques langues des États signataires. Par exemple, l'article 111 de la Charte des Nations Unies, signée le 26 juin 1945, reconnaît que les textes chinois, français, russe, anglais et espagnol font également foi. L'OCDE ou encore le Conseil de l'Europe ont opté pour un nombre plus restreint puisque seuls l'anglais et le français disposent du statut de langues officielles.

Par conséquent, il convient de souligner l'originalité du régime linguistique de l'Union européenne qui entraîne un lourd travail de traduction des textes contraignants à destination des États membres. Ainsi, la traduction du droit non territorialisée, créatrice d'une règle unique de droit international ${ }^{13}$, est une traduction verticale (1.1) dont la particularité réside sur le plan traductionnel dans l'équivalence «uniformisante» qu'elle sous-tend (1.2).

\subsection{Une traduction verticale}

À la différence d'autres organisations internationales qui ont fait le choix d'un bilinguisme ou d'un plurilinguisme limité, l'Union européenne a privilégié un cadre 
juridique garant de l'égalité des langues. Il est même souligné qu'au sein des institutions internationales, le choix des langues officielles relève plus d'une volonté de représentation politique que d'une recherche du caractère véhiculaire de la langue choisie (Barthe-Gay 2007: 141). Quoi qu'il en soit, seuls les textes des traités, dans leurs versions linguistiques officielles, font foi. Ainsi, dans la pratique, la négociation et la rédaction se font en deux ou trois langues par les représentants des États contractants; les autres textes authentiques sont, ensuite, traduits à partir des textes négociés (Barthe-Gay 2007: 143). Les pays dont la langue n'est pas reconnue comme langue officielle doivent se contenter d'une version dans une autre langue. La traduction qui en sera faite, en vue d'une application nationale, ne présentera aucune authenticité vis-à-vis de l'organisation. Par conséquent, il existe une situation inégalitaire entre les langues des États signataires d'une convention internationale.

Cela peut expliquer le fait que la règle posée, au sein de la Communauté économique européenne devenue l'Union européenne, mette sur un pied d'égalité toutes les langues des États membres. En effet, dès «l'origine, les auteurs des traités constitutifs des Communautés européennes ont fait ouvertement le choix du multilinguisme intégral reposant sur le principe de l'égalité entre toutes les langues officielles et écartant le recours à une langue unique» (Rideau 2007: 63). De même, ce principe a été repris dans les textes du droit dérivé qui régissent le fonctionnement des institutions $^{14}$. En vertu de l'article 1 du règlement 1/1958, toutes les langues officielles sont des langues de travail. Toutefois, dans la pratique des institutions, compte tenu du problème du multilinguisme, les institutions et les organes de l'Union européenne bénéficient d'un droit à l'auto-organisation de leur fonctionnement.

De façon succincte, au sein des principales institutions, il apparaît que le Conseil européen, le Parlement européen et le Conseil de l'Union européenne respectent le multilinguisme intégral. Par contre, le COREPER utilise trois langues de travail (l'anglais, le français et l'allemand). La PESC, ayant pour langues de travail l'anglais et le français, bénéficie d'un régime particulier. De même, la Commission européenne utilise également des langues de travail sans que cela soit prévu par le règlement intérieur. «Pour le collège des commissaires et les services, il s'agit de l'anglais, du français et, dans une moindre mesure, de l'allemand sans que le règlement intérieur se réfère à cette pratique» (Rideau 2007: 82). Quant à la Cour de justice, la langue de travail interne est le français.

En application du principe du multilinguisme intégral, la traduction verticale concerne, tout d'abord, la catégorie d'actes normatifs de l'Union classés dans la hiérarchie verticale des normes juridiques ${ }^{15}$. Il s'agit des instruments qui ont un caractère contraignant. Cela concerne, en premier lieu, le droit primaire, à savoir les textes fondamentaux de l'Union européenne et des Communautés européennes. Il s'agit des traités constitutifs (versions originaires et versions mises à jour), des traités modificateurs, des traités d'adhésion pour les six élargissements et d'autres textes de base. Mais la traduction verticale concerne, également, le droit dérivé, à savoir les actes de portée générale comme les règlements qui, directement applicables, s’imposent dans les ordres juridiques des États membres dès leur publication au Journal officiel. À ceux-ci s'ajoutent les directives qui ont une force contraignante de moins grande portée parce qu'elles ne font que lier le ou les États membres destinataires quant au résultat à atteindre. Enfin, le troisième type d'acte juridique contraignant du droit dérivé est la décision individuelle qui est obligatoire dans tous ses éléments, 
mais à destination des seuls destinataires désignés par celle-ci. En marge du droit dérivé, nous ajouterons la décision-cadre qui, au même titre que la directive, lie les États membres quant aux résultats à atteindre et laisse la liberté aux instances nationales de mettre en œuvre les moyens réalisant les objectifs fixés.

En outre, le multilinguisme constitue "un enjeu pour la démocratie européenne» (Legendre 2009: 8). Il assure l'égalité des droits des citoyens et des États membres de l'Union européenne dans l'accès à la législation communautaire et dans leurs relations avec les institutions. La traduction permet l'accessibilité linguistique du droit de l'Union européenne mise au service d'une transparence démocratique et d'une sécurité juridique. Le doublement du nombre des langues officielles de l'Union à la suite des élargissements successifs et l'inflation normative communautaire constituent un défi au principe du multilinguisme intégral.

En effet, les différents services de traduction des institutions et des organes de l'Union remplissent la tâche ardue de produire, dans les vingt-trois langues officielles, les actes normatifs qui entrent dans la hiérarchie verticale des normes juridiques. Comme le souligne Umberto Eco, par sa célèbre formule, la langue de l'Europe, c'est la traduction. Cette verticalité normative, qui en application du principe du multilinguisme intégral rend la traduction obligatoire, caractérise la traduction verticale. Afin de mener à bien ce travail de traduction, les méthodes utilisées sont diversifiées. La méthode classique consiste à faire traduire le texte dans la langue maternelle du traducteur. Les méthodes «bipolaire» et «tripolaire» se distinguent selon que la langue source est la langue maternelle du traducteur ou que ni la langue source ni la langue cible ne sont sa langue maternelle. Enfin, la traduction «relais» est une méthode qui permet à un premier traducteur de traduire le document dans une langue «relais» (généralement le français ou l'anglais) puis un autre traducteur traduit le document dans la langue demandée. Cette méthode est utilisée pour les combinaisons linguistiques rares (traduction de l'estonien vers le grec par exemple).

Les instruments de travail sont des outils informatiques d'aide à la traduction. Une base de données terminologique interinstitutionnelle (base IATE) a été constituée sous l'impulsion du Comité interinstitutionnel de la traduction et de l'interprétation. Les mémoires de traduction partagées et les systèmes de reconnaissance vocale apportent une aide supplémentaire.

Cet arsenal doit permettre d'énoncer un droit unique dans vingt-trois langues officielles mais, nonobstant cette variété linguistique, le droit s'exprime dans une langue internationalisée ${ }^{16}$. Celle-ci "a été développée de façon à devenir accessible à tout le monde et à n'importe qui, indépendamment de toute appartenance communautaire» (Puppo 2002: 23). Par conséquent, «la langue internationalisée, dont la traduction, si l'on combine certaines intuitions de Quine et MacIntyre, serait d'autant plus facile que son objet est dépourvu d'une signification socialement enracinée» (Puppo 2002: 31). L'appartenance commune au fonds commun constitue ce que Quine désigne sous l'expression «verbalisme interculturel»(Quine 1977: 122). Puppo prend pour exemple paradigmatique du verbalisme interculturel le discours juridique international fondé sur les droits de l'homme. "Tout le monde comprend mais personne ne sait. Les phrases en termes de droits des hommes peuvent être traduites sans problèmes d'une langue internationalisée à une autre mais cela n'implique pas qu'elles signifient quelque chose dans chacune des langues en usage» (Puppo 2002: 
30). Le droit unique n'exprime pas une tradition juridique mais combine les diverses cultures des États membres. Cependant, le résultat figé qu'énonce le droit unique marque une distance avec le contenu du droit positif des États membres. Par conséquent, la traduction s'en trouve facilitée.

En effet, le droit supranational traduit s'exprime dans une langue artificielle, technique et administrable ${ }^{17}$. Il s'agit d'une langue institutionnalisée qui dépouille le langage juridique de toute empreinte culturelle propre. Elle se distancie du référent auquel le terme renvoie dans la culture juridique de l'État membre.

Le choix terminologique consiste en une acceptabilité linguistique en vue d'une interprétation uniforme du droit supranational.

\subsection{Une équivalence "uniformisante»}

Les règles de traduction fixées, les outils informatiques mis en place, les glossaires établis et les mémoires de traduction éprouvées forment autant de gages d'homogénéité et d'efficacité de la traduction verticale. L'Union européenne procède à une transposition juridique et également linguistique afin d'éviter de compromettre l'interprétation du texte. Le droit de l'Union, une fois énoncé dans toutes ses vingttrois langues officielles, doit être appliqué et interprété de façon uniforme au sein des ordres juridiques des vingt-sept États membres. En 1969, par l'arrêt Stauder, la Cour de justice des Communautés européennes a clairement énoncé que la nécessité d'une application et, dès lors, d'une interprétation uniforme, exclut qu'une version soit considérée isolément mais exige une interprétation à la lumière des versions établies dans toutes les langues. Autrement dit, «il s'agit de l'expression multiple d'une même disposition et de son contenu notionnel défini par rapport à un unique ordre juridique, l'ordre communautaire» (Bauer-Bernet 1982: 190).

En outre, afin de faciliter l'interprétation uniforme du texte, chaque terme doit s'entendre «abstraction faite du détail des règles positives auquel il est associé dans un droit déterminé» (Glanert 2006: 1240). Des auteurs constatent que la langue cible se détache de sa propre culture afin de mieux exprimer le droit unique rendu par la traduction ${ }^{18}$. En son temps, le latin médiéval, lingua franca de l'Europe de jadis, n'offrait-il pas le modèle d'une langue détachée d'une culture qui, il est vrai, était morte et n'avait jamais ressuscité en tant que telle (Moréteau 1999: 154)?

Le multilinguisme intégral conduit en quelque sorte à créer un vocabulaire juridique idéal qui, pour reprendre la métaphore de Simone Glanert (2006: 1240), serait un «clonage juridique», c'est-à-dire une conception artificielle faite en «laboratoire», et qui serait ensuite reproduit à l'identique dans une multitude de langues différentes. Une sorte de «pasteurisation» terminologique pour reprendre l'expression de Mitchell Lasser (2005). Inévitablement, pendant la traduction et l'interprétation, quelque chose du sens originel se perd. Ceci vient également du caractère «négocié» du droit de l'Union européenne et, de façon générale, de la qualité rédactionnelle assez pauvre de celui-ci. "L'utilisation de néologismes est assez rare dans le droit de l'UE» car la création d'un «euro langage» ou «jargon européen» pour exprimer un nouveau concept serait mal ressentie. Habituellement, la pratique consiste plutôt à donner une nouvelle signification à des termes qui existent déjà (Piris 2005: 476-477).

Hélène Bauer-Bernet, directrice de la documentation juridique informatique au service juridique de la Commission des Communautés européennes, explique que 
pour éviter le risque de confusion entre l'acception nationale et l'acception communautaire d'un même mot, il est admis de créer une terminologie communautaire. Toutefois, cette latitude est utilisée avec discrétion et elle est tempérée par le respect des particularités linguistiques ou juridiques. Par exemple, il peut être renoncé à l'équivalence mot à mot dans l'intérêt de la clarté; ainsi, certaines versions se comprennent mieux par l'emploi d'une catégorie abstraite (par exemple en français «sociétés civiles et commerciales») et d'autres par une énumération casuistique (par exemple toutes les formes de sociétés civiles ou commerciales en anglais) (Bauer-Bernet 1982: 191-192).

Cela ne va pas sans poser de problème car si le droit de l'Union européenne est unique, les États destinataires sont bâtis sur des cultures juridiques différentes. "Les ordres juridiques de l'Union européenne et ceux des États membres, en principe indépendants les uns des autres, sont en réalité imbriqués. Ils ont en commun le fait de devoir être mis en œuvre sur un même territoire et pour, ou par, une même population» (Piris 2005: 477). En principe, l'idéal serait que chaque concept puisse être rendu, dans la même langue, au moyen de sa propre terminologie spécifique, distincte de toute autre terminologie exprimant un autre concept.

Dans le cas de l'ordre juridique de l'Union européenne, cette question se pose souvent. Elle est difficile à résoudre, faute d'un lien entre les significations différentes données à un même terme selon les pays. Il est ainsi plus facile pour un juriste français de comprendre qu'un même terme juridique français signifie autre chose en Belgique qu'en France. Il est plus facile de le comprendre, parce que les deux ordres juridiques ne s'appliquent pas sur le même territoire. Mais il est plus difficile de comprendre une telle différence lorsqu'il s'agit de l'utilisation d'un même terme dans l'ordre juridique français et dans celui de l'Union européenne, parce que les deux ordres juridiques s'appliquent sur un même territoire, à savoir la France. (Piris 2005: 477)

Nous relevons ici la prégnance du lien langue, droit et territoire sur laquelle nous reviendrons en conclusion.

En outre, les juristes-réviseurs ont la charge d'assurer la révision des textes traduits. Il ne s'agit pas de rapprocher d'un texte «original» les autres versions linguistiques, mais d'assurer de manière symétrique l'équivalence de toutes les versions; toute version peut donc être modifiée pour mieux exprimer la "ratio legis ${ }^{19}$, notamment en ce qui concerne les dispositions constitutives de droit (p. ex. suppression de la référence à «enfant reconnu» qui n'est pas une notion commune aux droits des États membres) (Bauer-Bernet 1982: 192).

Enfin, pour pallier une éventuelle difficulté d'interprétation uniforme, il n'est pas rare de voir insérer dans les instruments européens, au début d'un acte juridique, un article fournissant une définition précise de tous les concepts cités. Le sens donné au concept, ainsi introduit dans les vingt-trois langues officielles, marque le choix politique opéré par les institutions. L'équivalence posée par toutes les définitions «uniformisantes» du concept énoncé constitue la matérialisation linguistique du droit négocié. Le consensus traductif opéré par l'instrument de droit uniforme détache le signifié des cultures juridiques nationales des États membres dont il provient. En cas de litige sur une version linguistique, il est courant de voir un terme dans une langue communautaire recevoir, en droit communautaire par l'interprétation de la Cour de justice, une acception différente de l'acception en droit national ${ }^{20}$. 
Certains en concluent que «l'interaction voulue des droits en Europe passe par une interaction forcée des langues» (Glanert 2006: 1233).

L'ensemble de ces spécificités caractérise la traduction verticale réalisée au sein de l'Union européenne. Dans ce contexte, le traducteur se met au service d'une volonté institutionnelle terminologique. Qu'en est-il lorsque la traduction est territorialisée?

\section{La traduction du droit territorialisée}

Nous savons que la traduction concerne exclusivement deux langues, en tant que phénomène binaire (Ladmiral 2004: 26) et qu’elle est, par conséquent, "un fait de bilinguisme» (Mounin 63: 4). Lorsque la traduction porte sur un texte de droit, elle constitue, en outre, un fait de «bijuridisme». Il en résulte que le processus traductif du droit conduit à comparer le droit exprimé dans la langue source au droit cible, avant de restituer le texte juridique dans la langue du droit cible.

Les contextes et les situations juridiques de la traduction du droit réalisée au sein d'un État sont divers. En ce sens, la traduction territorialisée diffère de la traduction non territorialisée sur plusieurs plans. Tout d'abord, la traduction s'opère de manière horizontale parce qu'elle consiste à faire passer le sens d'un texte juridique entre deux droits et deux langues. La mise en contact s'établit dans un rapport d'égalité, au même titre que la traduction médiévale entre les langues vulgaires, et vise à la compréhension du contenu d'un droit exprimé dans une langue. Pour ce faire, la comparaison s'avère être le passage obligé, voire incontournable, de tout processus traductif. Cette opération présente d'autant plus de difficultés que le concept objet de la traduction est pourvu d'une signification socialement enracinée. Il s'agit de mettre en communication deux droits et deux langues empreints de leur culture d'origine, sans toutefois que ni l'un ni l'une ne domine l'autre. Il convient, par conséquent, de souligner les caractéristiques de la traduction horizontale (2.1) dont la particularité réside sur le plan traductionnel dans l'équivalence localisée qu'elle sous-tend (2.2).

\subsection{Une traduction horizontale}

La traduction juridique réalisée au sein d'un État, par des institutions ou des traducteurs indépendants, trouve ses justifications dans divers contextes de droit. Nous prendrons trois exemples.

Tout d'abord, la traduction juridique peut être effectuée pour des personnes de droit privé: des sociétés commerciales, des particuliers, afin de permettre l'application du droit. Dans ce cas, elle porte sur des contrats commerciaux, des documents administratifs ou des actes authentiques, et elle a pour fonction de permettre de faire valoir des droits ou de faire reconnaître une situation juridique par une administration d'un autre État: un mariage ou un divorce entre deux personnes de nationalité différente, l'héritage d'un bien situé à l'étranger, un contrat de travail rédigé en langue étrangère, etc.

Puis, la traduction peut intervenir dans un contexte scientifique et servir soit à connaître le droit étranger, soit à faire connaître son propre droit à l'étranger. Dans ce cas, elle porte sur des ouvrages doctrinaux mais également sur des textes normatifs (constitution, code, lois, etc.). Par exemple, les autorités publiques françaises ont 
fait traduire par Juriscope les codes français en langues anglaise et espagnole en vue de leur diffusion sur le site de Légifrance. En outre, la traduction d'ouvrages rédigés en langue étrangère permet la divulgation des travaux en science juridique. Par exemple, les travaux de Kelsen ont été, entre autres, traduits en français, anglais, tchèque, hongrois, polonais et japonais. Une illustration du fait que nous vivons dans un monde traduit mais également que nous travaillons dans une science traduite. Comme le souligne Rodolfo Sacco (2009), la traduction est au service de la connaissance du droit.

Enfin, la traduction juridique peut également être effectuée pour le compte de la justice. Dans ce cas, elle permet soit le dialogue entre les autorités judiciaires des États comme dans le cadre de la traduction d'un mandat d'arrêt européen ou d'une commission rogatoire internationale, soit le dialogue entre l'autorité judiciaire et le justiciable qui ne comprend pas la langue dans laquelle se déroule la procédure. La justesse terminologique, comme aboutissement du processus traductif, présente des enjeux notables en justice. "Or lorsqu'on sait par expérience qu'un seul mot est de nature à changer la face d'un procès, on perçoit l'acuité du problème» (Michaud 1985: 267). En effet, «bien des batailles juridiques, bien des décisions judiciaires tournent autour de mots, voire de traductions, plus qu'autour de faits» (Groffier et Reed 1990: 3).

Ces trois exemples illustrent la diversité des contextes juridiques de la traduction horizontale, ce qui diffère de l'unicité du contexte supranational de la traduction verticale. À la différence également, la traduction horizontale ne vise pas la création d'un droit unique mais la connaissance d'un droit autre. Par conséquent, l'une des difficultés de la traduction horizontale réside dans le transfert de sens d'un droit exprimé dans une langue et d'un langage propres empreints de leur culture d'origine vers une autre langue et un autre droit. Comme le souligne Jean-Claude Gémar (2002: 163), le texte juridique est sans doute l'un des plus marqués culturellement. «Le langage du droit véhicule des notions propres à une tradition, une culture et produit des textes le plus souvent porteurs de règles ou normes contraignantes et d'effets juridiques » (Gémar 1998: 7). Par conséquent, traduire un texte de droit reviendrait à raisonner plutôt en fonction des «cultures juridiques» (Chiba, Capeller, et al. 1998), des «traditions juridiques» (Glenn 2003: 276) et des «systèmes juridiques» (Legeais 2008).

Simeoni (2004: 72) souligne que « [c]ertains systèmes culturels, plus que d'autres, font bon accueil aux traductions, voire les sollicitent et souvent même les imitent, tandis que d'autres les rejettent. Surtout, la question du contact des langues est posée comme cas particulier de celle du contact des cultures».

Lorsque le comparatiste aborde un autre droit, «le contexte culturel tient évidemment une place majeure parce que l'observateur observe et compare à partir de ses références culturelles» (Mathieu-Izorche 2009: 130). Cependant ses références culturelles peuvent contribuer à son enfermement (Izorche 2001: 315).

Face à ce constat, la traduction du droit territorialisée sous-tend une équivalence traductionnelle localisée.

\subsection{Une équivalence localisée}

Il a été observé, tant par les juristes comparatistes que par les linguistes et traductologues, que le droit en tant que produit social d'une culture territorialisée oppose fréquemment une résistance à la traduction. 
Dans L'Épreuve de l'étranger, Berman explique que les cultures résistent à la traduction par pur réflexe ethnocentrique. «Des résistances intimes motivées par la peur, voire la haine de l'étranger, perçu comme une menace dirigée contre notre propre identité langagière» (Ricœur 2001 : 135). Derrière ces résistances de la traduction, ce refoulement de l'étranger, se profilerait un refus de l'altérité, une forme de narcissisme culturel et d'ethnocentrisme.

Lorsque le comparatiste observe et compare un autre droit, ses références culturelles risquent d'influencer son observation et «de l'entraîner vers les dérives de l'ethnocentrisme» (Mathieu-Izorche 2009: 130). Le comparatiste Olivier Moréteau (2004: 2) remarque que l'une des premières causes du retard du droit comparé en France est «à rechercher dans l'ethnocentrisme du juriste français ».

Le processus de traduction du droit de l'autre consiste à dominer l'opposition entre l'identité et l'altérité juridique. "L’altérisation de l'identité juridique étant asservie à une appartenance originaire, le façonnement d'un soi», lors de la comparaison des droits, présente des limites (Legrand 2006: 13), pour cause d'ethnocentrisme. Ne pourrait-on pas ici plutôt introduire le néologisme «juricentrisme»?

À l'instar de l'égocentrisme - hermétique à toute empathie - ou de l'ethnocentrisme - tendance à considérer son groupe social comme le seul modèle de référence -, le «juricentrisme» consiste, de manière inconsciente, à considérer son propre droit comme un modèle ramenant ainsi toute analyse des autres droits à ses propres critères. Cette démarche rend l'équivalence traductionnelle fortement localisée. Le «juricentrisme» ou l'ethnocentrisme du juriste conduit, d'une part, à percevoir toute différence du droit de l'autre par rapport à ce «modèle» comme un signe d'infériorité et, d'autre part, tend à ignorer, voire déprécier, les éléments différents de l'autre droit, sans en apprécier la pertinence. Cette attitude inconsciente varie suivant si le droit de l'autre émane d'un pays qui est considéré «digne» d'admiration ou non - comme le droit anglo-saxon -, qu'il s'agit d'une ancienne colonie ou pas, d'une puissance économique, ou que le droit est fondé sur des principes religieux ou philosophiques, etc. (Monjean-Decaudin 2006: 42).

Pour un traducteur, le «juricentrisme» consiste à traduire coûte que coûte, au détriment de la culture juridique source, un terme ou un concept sans équivalence par un terme ou concept propre à son droit et à sa langue. Il s'agit de tirer à soi le droit étranger pour l'amener vers la terminologie ou le concept de son propre système de pensée juridique, dénaturant à la fois le droit source et la traduction. L'équivalence ainsi établie porte une empreinte culturelle juridique localisée sans permettre un véritable transfert de sens. Thiry $(2000: 2)$ constate que cela est un défaut fréquent et préconise une «saine méthodologie». Il récuse toute idée de construire un schéma propre à un seul système notionnel-linguistique auquel l'on soumettrait ceux qu'on lui comparerait.

Il est souvent admis que l'équivalence semble d'autant plus envisageable dès lors qu'il existe une parenté, c'est-à-dire le partage d'un fonds, linguistique et juridique, commun entre deux langues et deux droits. Gémar (2002: 165) remarque que par une sorte d'intuition, l'on pense "que plus une culture est éloignée de celle du traducteur, plus celui-ci aura de la difficulté à la faire passer dans son texte d'arrivée». Aussi, la proximité ou parenté des droits et des langues tend à être considérée comme un facteur facilitant la comparaison-traduction. Alberto Puppo (2002: 22) désigne 
la parenté par le fonds commun, le patrimoine linguistique partagé entre deux langues, selon leur degré d'affinité.

Par conséquent, suivant le degré de parenté, le passage d'une langue à l'autre ou d'un droit à l'autre est rendu plus ou moins facile, la «frontière» qui les sépare est alors plus ou moins perméable et les échanges s'en trouvent alors plus ou moins fluidifiés. En général, une perméabilité fonctionnelle des concepts juridiques a déjà été établie en droit comparé et nous les identifions comme une sorte d' "universaux juridiques» (Pelage 2001: 77). Ils constituent un «minimum invariant» et correspondraient, en quelque sorte, au génotype avancé par le comparatiste Rodolfo Sacco ${ }^{21}$. Nous dénommons cette prédisposition génétique «zone de perméabilité». Elle se caractérise par une prédisposition interculturelle qui facilite les processus traductif et comparatif, autrement dit ceux-ci seraient «bémolisés» (Legrand 2006: 4) ${ }^{22}$.

Par exemple, l'Espagne et la France appartenant toutes deux à la famille des langues romanes et à la famille des droits romano-germaniques, les flux interlinguistique et interjuridique s'avèrent facilités grâce à cette zone de perméabilité entre droits et langues. Toutefois, il ne peut être conclu à une correspondance notionnelle et linguistique parfaite rendant l'équivalence automatique. Bien au contraire, une certaine méfiance doit être manifestée face à ce type de proximité qui tend à tromper la vigilance traductionnelle par les pièges que constituent les faux amis. Comme l'observe Gémar (2002: 165), il ne faut pas «sous-estimer les obstacles que présentent les langues et cultures "germaines" 23 ».

Picard (1999: 894) considère que «le comparatiste se présente comme une sorte de traducteur». Il souligne que la traduction suppose que l'une et l'autre langues comportent toutes deux des signifiants désignant ou exprimant des signifiés identiques ou au moins équivalents, ce qui est loin d'être acquis compte tenu qu' "en droit, si des mots équivalents existent, l'équivalence n'est qu'apparente ou très approximative, quand elle n'est pas trompeuse. Car derrière le mot, le concept juridique ne présente pas nécessairement la même consistance dans un ordre juridique et dans l'autre» (Picard 1999: 159). La nature de l'équivalence doit s'apprécier à l'aune du droit, de la langue, de l'histoire, de la société à un moment donné, etc.

En outre, l'équivalence est perçue de manière différente en langue et en droit.

Le problème d'équivalence juridique du texte traduit par rapport à l'original ne se pose pas dans les mêmes termes pour le traducteur et pour le juriste, le premier cherchant en principe à produire un texte équivalent et le second une équivalence juridique. Or, dans un cas comme dans l'autre, c'est la rencontre et la fusion harmonieuses des deux éléments constitutifs du texte - contenant et contenu - qui produiront l'équivalence souhaitable. [...] Peut-on, en traduction juridique, atteindre les deux objectifs à la fois, soit l'équivalence des textes dans chaque langue et dans chaque système, sans sacrifier l'un ou l'autre? (Gémar 2002: 171)

Il a été remarqué que «le droit refuse par définition qu’un texte puisse avoir des sens multiples, la normativité imposant l'univocité. Dès lors, le juriste ne se pose même pas la question» (Lamèthe et Moréteau 2006: 335). En effet,

parce que la langue dans laquelle le savoir juridique est exprimé et véhiculé n'est pas formalisée, parce qu'elle dépend d'une culture, d'une formation, d'une histoire, une question préalable se pose. Les termes utilisés pour désigner les notions et principes, voire pour les définir, ont-ils un sens fixé, un sens vrai à respecter pour en user 
correctement? [...] L'hypothèse primordiale est sans doute que la plupart des mots employés par les juristes ont, en général, un sens déterminé, clair, maîtrisé. (Atias 2002 : 84 et 88 )

Traduire le droit devient alors une tâche ardue car le droit n'est pas une science exacte. Comprendre le discours juridique renvoie «à cette distance qui sépare le dire du sens dont il est investi, le locutoire et l'illocutoire si l'on reprend la terminologie d'Austin» (Sueur 2001: 146). «Le traducteur, généralement seul devant son texte, ne peut compter que sur ses propres ressources pour atteindre l'équivalence recherchée. Or, en traduction juridique comme en traduction tout court, cette équivalence reste pour le moins aléatoire» (Gémar 2002: 174). Aléatoire dans le sens où le passage d'une langue et d'un droit sources à une langue et à un droit cibles devient hasardeux mais également problématique. Le tout étant de tenter de se prémunir contre toute tentation de juricentrisme.

Compte tenu de la diversité des contextes et des situations juridiques dans lesquels la traduction horizontale du droit prend sa source, il est manifeste que l'équivalence devient inhérente à l'objectif qui lui est assigné. Par exemple, en matière d'entraide judiciaire, le juge qui relève la déficience de la traduction considère néanmoins qu'elle est valable dans la mesure où l'objectif de la traduction dans la procédure est, tout de même, atteint. Ainsi, la Cour de cassation a considéré que cela ne portait pas grief, au motif que «même si les traductions d'une demande d'extradition qui ont été communiquées sont imparfaites, elles suffisent à apprécier le bien-fondé de la demande ${ }^{24} »$. Dès lors, les critères de l'équivalence échappent au champ de la linguistique et de la traductologie et relèvent d'un pouvoir souverain.

\section{Conclusion}

Le pouvoir quel qu'il soit, pèse dans le rapport entre langues et droits mais également entre diversité d'espaces et pluralité linguistique. Nous ne ferons qu'effleurer ici le sujet dont une analyse approfondie s'avérerait riche d'enseignements. Le territoire en tant qu'espace organisé est contrôlé par le droit et par la langue: «nommer/normer les lieux revient à s'approprier l'espace, à le dominer»(Guillorel et Koubi 1999: 7). Lorsque l'on traduit ne part-on pas d'une langue de départ pour atteindre une langue d'arrivée? Ce transport translinguistique ne correspond-il pas au passage de «frontières»? "Le territoire est à la fois le vecteur fondamental de la représentation du monde et l'instrument de son contrôle politique» (Guillorel et Koubi 1999: 7). Par conséquent, s'approprier la traduction reviendrait à user d'un hégémonisme linguistique afin de dominer un ou des territoires. Le droit mis au service du pouvoir imposerait la langue de traduction.

Prenons pour exemple l'anglais, largement utilisé dans les contextes de droit international. Actuellement, «la globalisation économique s'accompagne d'une globalisation linguistique. Il y a un risque d'hégémonie de l'anglais, même si chaque langue se défend» (Breton 2004: 2). L'indiscutable suprématie de l'anglais risque d'entraîner une uniformisation culturelle (Breton 2000: 24), donc un appauvrissement du patrimoine culturel mondial (Breton 2004: 1). De même, au sein de l'Union européenne, qui consacre pourtant le principe du multilinguisme intégral, l'on constate que si les langues officielles se multiplient, officieusement, l'anglais est de plus en plus privilégié (Scheidhauer 2008: 125). 
Sous les apparences d'une égalité entre toutes les langues officielles se profile en arrière-plan une préférence croissante pour l'unicité linguistique. Un rapport du Sénat pointe la multiplication des entorses au principe du multilinguisme. Par exemple, les conférences intergouvernementales d'adhésion se déroulent exclusivement en anglais, y compris par les représentants des pays membres ou observateurs de l'Organisation internationale de la francophonie ${ }^{25}$. De même, il n'existe pas de régime linguistique type concernant la quarantaine d'agences et d'organismes communautaires annexes. "L'absence de règles linguistiques harmonisées empêche dès lors de garantir une véritable diversité culturelle au sein de ces organes qui s'avèrent pourtant de plus en plus étroitement associés au processus décisionnel de l'Union européenne » (Legendre 2009: 33). Le rapporteur Legendre met en garde contre le glissement vers l'unilinguisme et le risque subséquent de la généralisation d'un système de pensée unique, en ces termes:

En effet, lorsqu'un intervenant se voit contraint, pour des raisons principalement d'ordre logistique, à prononcer un discours dans une langue qui lui est étrangère, il est conduit à se placer sur le «terrain de l'autre». Dans le cas où il ne disposerait pas d'une maîtrise parfaite de la langue de ses interlocuteurs, son message est susceptible de perdre de son pouvoir de conviction. Même dans le cas où il serait parfaitement bilingue, il y a lieu de penser que son message serait nécessairement influencé par un cadre d'analyse juridique ou économique propre à la langue étrangère concernée, ce qui contribue par la même occasion à généraliser un système de pensée unique. La langue ne se résume pas, en effet, à un simple instrument de communication, elle est également le vecteur d'expression de cultures politiques, juridiques et économiques spécifiques aux pays dont elle est la langue naturelle. (Legendre 2009: 47)

Glanert souligne l'obstacle linguistique que constitue l'usage d'une langue de travail unique pour exprimer la diversité des droits des interlocuteurs. Elle se demande comment un juriste espagnol ou néerlandais peut expliquer fidèlement son propre droit en anglais. Et comment le juriste néerlandais pourrait dialoguer avec le juriste espagnol lorsque le droit espagnol est rendu à travers les limites intrinsèques à la langue anglaise. Elle en conclut que «le phénomène de transit obligatoire par la langue de travail ne pourra qu'entraîner pour les droits en présence d'irréversibles mutations signifiantes», car la "capacité de représentation de l'anglais - comme de toute langue d'ailleurs - est limitée, ce qui implique que les divers intervenants anglophones doivent admettre d'inévitables accommodements linguistiques» (Glanert 2005: 5).

Cette perte d'identité au travers d'un discours uniforme reviendrait à abandonner une part de son territoire et de son pouvoir. La mondialisation ne constitue-t-elle pas une sorte de mainmise territoriale et, partant, linguistique?

Ces quelques réflexions sur la territorialité ou l'extraterritorialité d'une traduction mettent en relief les interférences entre droit, langue, territoires et pouvoirs; des paramètres auxquels la traduction du droit est fortement liée.

Partagée entre trop (traduction territorialisée) et trop peu (traduction non territorialisée) de référent culturel, la traduction du droit présente des enjeux territoriaux et extraterritoriaux du fait de la mondialisation. L'équivalence traductionnelle n'est-elle pas en passe de devenir un objet et un sujet de convoitise dans les années à venir? 


\section{REMERCIEMENTS}

L'auteur tient à vivement remercier Jean-René Ladmiral et Nicolas Froeliger pour l'intérêt qu'ils ont montré à l'égard de cette contribution transversale.

\section{NOTES}

1. Suivant la théorie de De Saussure, «la langue est un produit social en ce sens que l'individu l'enregistre passivement» cité par Dubois, Giacomo, et al. (2001: 267); voir De Saussure, Ferdinand (1984): Cours de linguistique générale, Paris, Payot, p. 23 et s. et spéc. p. 25: «Mais qu'est-ce que la langue? Pour nous elle ne se confond pas avec le langage; elle n'en est qu'une partie déterminée, essentielle, il est vrai. C'est à la fois un produit social de la faculté du langage et un ensemble de conventions nécessaires, adoptées par le corps social pour permettre l'exercice de cette faculté chez les individus».

2. Le rapport entre langue et culture relève de l'ethnolinguistique. Selon l'hypothèse de Sapir-Whorf, la différence de langue a pour conséquence une structuration intellectuelle et affective différente; il s'agit ainsi de deux mondes différents et non pas du même monde sous deux séries d'étiquettes différentes. L'hypothèse, poussée à l'extrême, amène Whorf à supposer, par exemple, qu'un peuple dont la langue ignore la catégorie du temps grammatical vit dans un éternel présent. Sur les théories de Sapir et Whorf, voir Gémar (1995: 144).

3. Pour une définition de la règle de droit, voir Aubert (2007: 7-25).

4. À noter l'atlas de Georg Wenker, l'Atlas linguistique de la France de Jules Gilliéron et Edmond Edmont, ou encore l'Atlas des langues du monde de Roland Breton.

5. Cette classification a été établie dès 1964 par René David dans son ouvrage «Les grands systèmes de droit contemporains".

6. Legeais (2008: 89) mentionne que René David fonde son raisonnement sur le fait qu'il existe des systèmes de droit qui reposent sur une même technique, mais qui ont une signification et une portée sociale tellement différentes qu'il serait absurde de les réunir dans une même famille: ainsi la conception de l'État et du droit mis en œuvre en URSS s'éloignait tant de la conception française, que les systèmes de droit respectifs des deux pays, bien que techniquement fondés avant tout sur la loi, ne pouvaient qu'être retenus dans des familles différentes.

7. Voir par exemple, Wald (2005: 187-197).

8. À noter qu'il existe plusieurs typologies des universaux et que la recherche des universaux du langage a pris une particulière acuité dans les années 60 , sous la pression de deux types de recherches: les recherches concernant la traduction automatique et la théorie des grammaires génératives.

9. Voir les ouvrages qui s'y réfèrent comme ceux de Tunc (1970), de David et Jauffret-Spinozi (2002), de Fromont (2001) et de Legeais (2008).

10. Sur le flux et le reflux du colonialisme, voir Legeais (2008: 59). Selon l'auteur, il semble bien que le Japon soit maintenant un modèle pour la Corée du Sud.

11. Voir également Bertolucci Pizzorusso (2003: 6) et les études menées sur l'Histoire des traductions vers la langue française au Moyen Âge.

12. La proposition de Robert Schuman figure dans la Préface de l'ouvrage de Reuter (1953).

13. Il s'agit en fait d'un droit régional.

14. Le règlement linguistique de la CEE du 15 avril 1958, JO CE 6 octobre 1958, p. 285/58, modifié à plusieurs reprises, et notamment par le règlement (CE) no 920/2005 du Conseil du 13 juin 2005, JOUE, $n^{\circ}$ L 156 du 18 juin 2005, qui pose le principe de l'égalité entre les langues officielles.

15. Pour la typologie des actes et la hiérarchie des normes dans l'UE, v. Résolution du Parlement européen sur la typologie des actes et la hiérarchie des normes dans l'Union européenne (2002/2140(INI)), JOUE, C 31, du 5 fév. 2002, p. 126-134.

16. La distinction faite entre langues en usage et langues internationalisées a été élaborée par MacIntyre (1993: 401).

17. Jori (1995: 119) soutient, à partir de la distinction entre langues naturelles et langages artificiels, que les acteurs du droit positif se servent d'un langage administré.

18. Notamment Glanert (2006) et Bauer-Bernet (1982).

19. Expression latine signifiant «raison de la loi», utilisée pour désigner la raison d'être de la règle établie d'où l'interprétation tire la mesure de la pleine application de celle-ci.

20. Par exemple «salaire», «entreprise», « détournement de pouvoir », voir Hélène Bauer-Bernet (1982: 192). 
21. Le «génotype» est une idée primordiale qui peut être trouvée dans un ensemble de systèmes juridiques similaires. Le "phénotype» représente la concrétisation ou la réalisation adoptée par le génotype dans tel ou tel système.

22. L'auteur considère que dans le cas de droits d'une même tradition juridique, la comparaison s'en trouve bémolisée, c'est-à-dire facilitée.

23. Voir également Sparer (1979).

24. Cour de cassation, Chambre criminelle, 2 septembre 1997, n 97-83.256, non publié.

25. L'Union européenne compte 15 États membres appartenant à la Francophonie.

\section{RÉFÉRENCES}

Atias, Christian (2002): Épistémologie juridique. Paris: Dalloz.

Aubert, Jean-Luc (2007): Introduction au droit. Paris: PUF.

BARTHE-GAY, Clarisse (2007): Interprétation et traduction dans le droit des traités internationaux. In: Jean-Jacques Sueur, dir. Interpréter et traduire. Bruxelles: Bruylant, 139-150.

Bauer-Bernet, Hélène (1982): Le multilinguisme du droit de la Communauté européenne. In: Jean-Claude GÉmar, dir. Langage du droit et traduction. Essais de Jurilinguistique. Montréal: Linguatech et Conseil de la langue française, 187-197.

Bergel, Jean-Louis (2004): Théorie générale du droit. Paris: Dalloz.

Berman, Antoine (1995): L'épreuve de l'étranger. Paris: Gallimard.

Bertolucci Pizzorusso, Valeria (2003): La réception de la littérature courtoise du $\mathrm{XII}^{\mathrm{e}}$ au XIV ${ }^{e}$ siècle en Italie: nouvelles propositions. In: Barbara K. Altmann et Carleton W. CArroll, dir. The Court Reconvenes: Courtly Literature Across the Disciplines. Selected Papers from the Ninth Triennial Congress of the International Courtly Literature Society (Vancouver, 25-31 juillet 1998), 3-13.

BREton, Roland (2000): La guerre des langues, La suprématie de l'anglais est-elle inéluctable? Le Courrier de l'UNESCO, Dossier Guerre et paix des langues, 23-25.

Breton, Roland (2008): Atlas des minorités dans le monde. Panorama des identités ethniques et culturelles. Paris: Autrement.

Breton, Roland et MAzoyer, Krystyna (2004): Atlas des langues du monde: Une pluralité fragile. Paris: Autrement.

Chiba, Masaji, Capeller, Wanda et Kitamura, Takanori (1998): Une introduction aux cultures juridiques non occidentales. Bruxelles: Bruylant.

Cornu, Gérard (2005): Linguistique juridique. Paris: Montchrestien.

Connu, Gérard (2007): Vocabulaire juridique. Paris: PUF.

DAVID, René (1964): Les grands systèmes de droit contemporains. Paris: Dalloz.

DAVid, René et JAUfFret-Spinosi, Camille (2002): Les grands systèmes de droit contemporains. Paris: Dalloz.

Dubois, Jean, Giacomo, Mathée, Guespin, Louis et al. (2001): Dictionnaire de linguistique. Paris: Larousse.

Folena, Gianfranco (1991): Volgarizzare e tradurre. Turin: Einaudi.

Fromont, Michel (2001): Grands systèmes de droit étranger. Paris: Dalloz.

GÉMAR, Jean-Claude (1995): Le langage du droit au risque de la traduction. In: Gérard Snow et Jacques Vanderlinden, dir. Français juridique et science $d u$ droit, Bruxelles: Bruylant, 124-154.

GÉmAR, Jean-Claude (1998): Les enjeux de la traduction juridique, principes et nuances. Traduction de textes juridiques: problèmes et méthodes, Equivalences 98, Séminaire ASTTI du 25 septembre 1998. Consultée le 25 octobre 2010, <http://www.tradulex.org/Actes1998/ Gemar.pdf>.

GÉmAR, Jean-Claude (2002): Le plus et le moins-disant culturel du texte juridique. Langue, culture et équivalence. Meta. 47(2):163-176.

GLANERT, Simone (2006): La langue en héritage: réflexions sur l'uniformisation des droits en Europe. Revue internationale de droit comparé. 4:1231-1247. 
Glenn, H. Patrick (2003): La tradition juridique nationale. Revue internationale de droit comparé. 2:276-278.

Groffier, Ethel et Reed, David (1990): La lexicographie juridique, principes et méthodes. Québec: Yvon Blais.

Guillorel, Hervé et Koubi, Geneviève (1999): Introduction. In: Hervé Guillorel et Geneviève KoubI, dir. Langues \& Droits, Langues du droit, droit des langues. Bruxelles: Bruylant, 7-29.

Izorche, Marie-Laure (2001): Propositions méthodologiques pour la comparaison. Revue internationale de droit comparé. 53(2):289-325.

JORI, Mario (1995): Definizioni giuridiche e pragmatica. Analisis e diritto, 109-144.

LADMIRAL, Jean-René (2004): Dichotomies traductologiques. La linguistique. 40(1):25-50.

Lamèthe, Didier et Moréteau, Olivier (2006): L'interprétation des textes juridiques rédigés dans plus d'une langue. Revue internationale de droit comparé. 58(2):327-362.

Lasser, Mitchel (2005): The European Pasteurization of French Law. Cornell Law Review. 90:995-1083.

LegeAIS, René (2008): Grands systèmes de droits contemporains: une approche comparative. Paris: Litec.

Legendre, Jean (2009): Rapport du Sénat $n^{\circ} 258$ (2008-2009), annexe au procès-verbal de la séance du 11 mars 2009. Consultée le 25 octobre 2010, <http://www.senat.fr/rap/108-258/ 108-2580.html $>$.

LegRAND, Pierre (2006): Le droit comparé, Paris: PUF.

Levi-Strauss, Claude (1958): Anthropologie structurale. Paris: Plon.

MacIntyre, Alasdair (1993): Quelle Justice? Quelle rationalité? (Traduit par Michèle Vignaux D'Hollande). Paris: PUF.

Mathieu-Izorche, Marie-Laure (2009): Approches épistémologiques de la comparaison des droits. In: Pierre Legrand, dir., Comparer Les Droits, résolument. Paris: PUF, 123-146.

Michaud, Jean (1985): Le traducteur et l'expert. Revue de science criminelle, $1^{\text {er }}$ semestre, 265269.

Monjean-Decaudin, Sylvie (2006): Rapport de stage. Master II Juriste linguiste, sous la direction du Professeur D. Breillat, Université de Poitiers.

Moréteau, Olivier (1999): L'anglais pourrait-il devenir la langue juridique commune en Europe? In: Rodolfo SACCo et Luca CASTELlani, dir. Les multiples langues du droit européen uniforme. Turin: ISAIDAT / L'Harmattan, 143-162.

Mounin, Georges (1963): Les problèmes théoriques de la traduction. Paris: Gallimard.

Pelage, Jacques (2001): Éléments de traductologie juridique: application aux langues romanes. Paris: Launay.

PiCARD, Etienne (1999): L'état du droit comparé en France, en 1999. Revue internationale de droit comparé. 51(4):885-915.

Piris, Jean-Claude (2005): Union européenne: comment rédiger une législation de qualité dans 20 langues et pour 25 États. Revue du droit public et de la science politique en France et à l'Étranger. 2:475-495.

Puppo, Alberto (2002): Les langues entre traditions et droit: de la traduction radicale au verbalisme interculturel. Revue semestrielle d'anthropologie et d'histoire. 44(2):22-31.

Quine, Willard Van Orman (1977): Le mot et la chose. (Traduit par Joseph Dopp et Paul Gochet). Paris: Flammarion.

Reuter, Paul (1953): La Communauté européenne Charbon-acier. Paris: LGDJ.

Riceeur, Paul (2001): Le juste 2. Paris: Éd. Esprit.

RideAu, Jacques (2007): L'Union européenne face aux défis linguistiques. In: Jean-Jacques SuEUR, dir. Interpréter et traduire. Bruxelles: Bruylant, 63-137.

SACco, Rodolfo (2009): Aperçus historique et philosophique des relations entre droit et traduction. In: Marie CoRnu et Isabelle De LAMBerTerie, dir. Droit de la traduction et traduction $d u$ droit. Actes du colloque international, 15 et 16 octobre 2009, Faculté de Droit de Poitiers, à paraître, vidéo consultée le 5 juin 2010, <http://uptv.univ-poitiers.fr/web/canal/61/ theme/29/manif/229/video/2013/index.html $>$. 
Scheidhauer, Christophe (2008): Les langues de l'Europe, un régime paradoxalement durable. Langage \& société. 125:125-143.

Simeoni, Daniel (2004): La langue de traduction. La linguistique. 1(40):67-82.

SPARER, Michel (1979) : Pour une dimension culturelle de la traduction juridique. Meta. 24(1):6894.

Sueur, Jean-Jacques (2001): Une introduction à la théorie du droit. Paris: L'Harmattan.

Terracini, Benvenuto (1957): Conflitti di lingua e di cultura. Venezia: Neri Pozza.

TERrÉ, François (1986): Brèves notes sur les problèmes de la traduction juridique. Revue internationale de droit comparé. 38(2):347-350.

Terré, François (2006): Introduction générale au droit. Paris: Dalloz.

THIRY, Bernard (2000) : Équivalence bilingue en traduction et en terminologie juridique: Qu'estce que traduire en droit? In: La traduction juridique: histoire, théorie(s) et pratique, actes du colloque international de Genève des 17, 18 et 19 février 2000. Consultée le 11 mai 2008, $<$ http://www.infotheque.info/cache/9601/www.tradulex.org/Actes2000/sommaire.htm>.

Toury, Gideon (1995): Descriptive Translation Studies and Beyond. Amsterdam/Philadelphia: John Benjamins.

Tunc, André (1970): Cours de grands systèmes de droit contemporain. Paris: Les cours de droit. WALd, Arnoldo (2005) : Doit-on repenser les familles juridiques? In: De tous horizons - Mélanges Xavier Blanc-Jouvan. Paris: Société de Législation Comparée, 187-197. 